\title{
Deferoxamine enhances cell migration and invasion through promotion of HIF-1 $\alpha$ expression and epithelial-mesenchymal transition in colorectal cancer
}

\author{
WENJING ZHANG, YAO WU, QINGQING YAN, FENG MA, XINPENG SHI, \\ YINGYING ZHAO, YING PENG, JIDE WANG and BO JIANG
}

Guangdong Provincial Key Laboratory of Gastroenterology, Department of Gastroenterology, Nanfang Hospital, Southern Medical University, Guangzhou, Guangdong 510515, P.R. China

Received September 4, 2013; Accepted October 7, 2013

DOI: $10.3892 / o r .2013 .2828$

\begin{abstract}
Deferoxamine (DFX), a metal chelator, has been previously reported to induce hypoxia and hypoxia-inducible factor- $1 \alpha$ (HIF-1 $\alpha)$ expression. HIF-1 $\alpha$ is a common inducer of epithelial-mesenchymal transition (EMT) in many solid tumors. However, the effect of DFX on cancer metastasis and the related mechanisms are not well established. In the present study, we aimed to ascertain whether DFX enhances EMT and cancer metastasis in colorectal cancer. After confirmation of DFX-inducing HIF-1 $\alpha$ expression, we examined the effect of DFX on cell adhesion, migration and invasion abilities and found a positive effect on the above functions. Consequently, cell morphology, cell growth and expression of EMT markers were assessed in cells with or without DFX treatment. We found that cells exposed to DFX were more isolated. They were spindle-shaped and looked similar to fibroblast-like cells, accompanied by increased anchorage-independent growth DFX-treated cells expressed E-cadherin and plakoglobin at a higher level, and vimentin and $\mathrm{N}$-cadherin at a lower level, when compared with these levels in control cells. Furthermore, the expression of E-cadherin in the cell membrane was markedly decreased in DFX-treated cells. These results suggest that DFX promotes cancer migration and invasion via a process consistent with EMT in colorectal cancer.
\end{abstract}

\section{Introduction}

Mounting evidence indicates that iron plays a crucial role in malignant cell proliferation $(1,2)$. A dysregulated iron metabo-

Correspondence to: Professor Bo Jiang or Professor Jide Wang, Guangdong Provincial Key Laboratory of Gastroenterology, Department of Gastroenterology, Nanfang Hospital, Southern Medical University, North Guangzhou Blvd. 1838, Guangzhou, Guangdong 510515, P.R. China

E-mail: drjiang@163.com

E-mail: jidewang@gmail.com

Key words: hypoxia-inducible factor- $1 \alpha$, deferoxamine, epithelialmesenchymal transition lism reveals a poor outcome in breast cancer and esophageal adenocarcinoma patients $(3,4)$. Deferoxamine (DFX), a high affinity iron chelator, is known to upregulate inflammatory mediators and it has been shown to have significant and selective antitumor activity, although the mechanisms of action remain to be completely elucidated (5-7). DFX has the ability of hypoxia mimetics to induce a hypoxic state which results in the upregulation or stabilization of hypoxia-inducible factor-1 (HIF-1) $(8,9)$. HIF-1 is a nuclear protein involved in the transcriptional activation of a broad array of genes that activate mitogenic, pro-invasive, pro-angiogenic and pro-metastatic genes (10-12). Hence, DFX-induced hypoxia is a complicated event, which may affect various pathways and gene expression through the regulation of HIF-1 expression.

Epithelial-mesenchymal transition (EMT) is a critical process for tumor invasion and metastasis (13-16). It is characterized by loss of cell polarity and cell adhesion, repression of E-cadherin expression and increased cell motility. At the same time, the traits of epithelial cells such as long-lasting morphological and molecular changes are altered to mesenchymal characteristics, such as fibroblastic morphology, increased vimentin and $\mathrm{N}$-cadherin expression, enhanced migration and invasion capacities, thus causing cancer cell metastasis $(14,17)$. It is important to note that the stabilization and activation of HIF-1 are the most important mechanisms that promotes metastasis and thereby, increase tumor aggressiveness $(18,19)$. For example, hypoxia or HIF-1 overexpression induces EMT and metastatic phenotypes through the direct activation of Twist in head and neck squamous cell carcinoma (HNSCC) (20). Hypoxia-induced EMT may be enhanced by the formation of the HIF-1/ $\beta$-catenin complex, which increases the transcriptional activity of HIF- $1 \alpha$ and consequently facilitates EMT under hypoxic conditions in hepatocellular carcinoma (HCC) (21).

In the present study, we treated colorectal cancer (CRC) cells with DFX to create a DFX-induced hypoxic cell model, and then explored EMT-associated features, such as the expression of EMT-associated molecules, the cellular location of E-cadherin and vimentin, cell morphological changes, adhesion, migration and invasion abilities. Our study showed that DFX treatment increased HIF-1 $\alpha$ expression and enhanced 
EMT. Thus, we reported the potential mechanism underlying DFX-induced hypoxia on cell migration and invasion in CRC cells.

\section{Materials and methods}

Cell culture and cell treatments. HT29 and HCT116 cells were grown in RPMI-1640 (Gibco-BRL) supplemented with 10\% fetal bovine serum (FBS) as we previously described (22). For DFX treatments, we utilized concentrations of $0,50,100$ and $200 \mu \mathrm{M}$ respectively, of DFX supplemented with 10\% FBS. Forty-eight hours later, cell morphological changes were observed or cell lysates were extracted to perform western blot analysis.

Western blotting, immunofluorescence assays and antibodies. Western blotting and immunofluorescence assays were carried out as previously described (23). Antibodies to HIF-1 $\alpha$ (Novus Biologicals, Littleton, CO, USA), E-cadherin and vimentin were applied in the immunofluorescence staining (Santa Cruz Biotechnology, Inc., Santa Cruz, CA, USA). Vimentin (prediluted), plakoglobin, N-cadherin and glyceraldehyde3-phosphate dehydrogenase (Abcam, Cambridge, UK) were all commercial products.

Hematoxylin and eosin $(H \& E)$ staining. Exponentially growing cells were seeded in coverslips and then exposed to $100 \mu \mathrm{M}$ of DFX. Forty-eight hours later, coverslips were fixed in $4 \%$ paraformaldehyde for $15 \mathrm{~min}$, washed in phosphate-buffered saline (PBS) and running water, followed by incubation with hematoxylin solution for $5 \mathrm{~min}$ and washed under running water. Subsequently, the coverslips were dipped in acid-ethanol, washed again, and then stained in eosin-ethanol (1\% eosin in $80 \%$ ethanol) for $3 \mathrm{~min}$, subjected to sequential dehydration and mounted. The coverslips were observed under an Olympus microscope (Olympus Optical Co., Ltd., Tokyo, Japan).

Immunofluorescence. Cells growing on coverslips were fixed with $4 \%$ paraformaldehyde at room temperature for $15 \mathrm{~min}$, washed with PBS and permeabilized with $0.1 \%$ Triton X-100 for $5 \mathrm{~min}$. After blocking with $1 \%$ bovine serum albumin (BSA) for $30 \mathrm{~min}$, cells were incubated with E-cadherin and vimentin antibodies for $2 \mathrm{~h}$. After washing with PBS, IgG Texas Red- (TR-) and IgG-FITC-labeled secondary antibodies were added and incubated for another $1 \mathrm{~h}$. Cells were then rinsed with PBS followed by Hoechst 33258 staining to reveal nuclei. Fluorescence images were captured and analyzed using the Olympus CKX41 fluorescence microscope (Olympus Optical Co., Ltd.).

Adhesion assay. Substrates for adhesion assays included $25 \mu \mathrm{g} / \mathrm{ml}$ fibronectin or $10 \mathrm{mg} / \mathrm{ml}$ heat-inactivated BSA. After overnight coating, wells were rinsed and blocked with $10 \mathrm{mg} / \mathrm{ml}$ heat-inactivated BSA as the negative control. Cells were treated with or without $100 \mu \mathrm{M}$ DFX for $48 \mathrm{~h}$, harvested, resuspended in serum-free medium, and $5 \times 10^{4}$ live cells were seeded into single wells of a 96-well plate. Cells were then incubated for $2 \mathrm{~h}$ at $37^{\circ} \mathrm{C}$. Subsequently, the detached cells were washed away, and attached cells were determine by MTT using a microplate reader.
Migration, invasion and wound healing assays. Cell migration and invasion assays were performed using Transwell inserts (BD Biosciences, Bedford, MA, USA) in a similar manner. The difference was that the upper surface of the chambers in the invasion assay was pre-coated with Matrigel (BD Biosciences), while not in the migration assay. Standard protocol was followed. In brief, cells were pre-treated with or without $100 \mu \mathrm{M} \mathrm{DFX}$ for $24 \mathrm{~h}$. Then cells were digested, washed and seeded into the inner chamber $(5,000$ cells/chamber) in medium without FBS. RPMI-1640 plus 15\% FBS was added to the lower chamber, and the plate was incubated for $24 \mathrm{~h}$. Cells on the upper surface of the filter were scraped away with a rubber scraper, while cells on the lower surface were fixed with $4 \%$ paraformaldehyde and stained with $0.5 \%$ crystal violet, and then counted under a light microscope. For the wound healing assay, DFX-treated cells were seeded in 6-well plates. After scratching the monolayer, cells were washed with PBS, cultured in RPMI-1640 plus 10\% FBS, and photographed under a x10 objective len at the indicated time points. All of the above assays were carried out in duplicate, and the results presented are the means of 5 random fields from each well.

Cell growth in soft agar. Cells that were treated with or without DFX $(100 \mu \mathrm{M})$ were harvested and pipetted well to become a single-cell suspension, and then mixed with regular medium containing $0.7 \%$ agar resulting in a final agar concentration of $0.35 \%$. The above cell suspensions were immediately plated in 6 -well plates coated with $0.6 \%$ agar in regular medium and cultured for 14 days. Tumor cell colonies were observed and counted under a dissecting microscope.

Statistical methods. Statistical analysis was carried out using SPSS 13.0 for Windows (SPSS, Inc.). Quantitative variables were analyzed using the $t$-test. $\mathrm{P}<0.05$ was considered to indicate a statistically significant result.

\section{Results}

DFX treatment induces HIF-1 $\alpha$ expression. To select a suitable cell candidate to induce HIF-1 $\alpha$ expression and EMT, we first detected the endogenous expression level of HIF-1 $\alpha$ in 6 colorectal cancer cell lines. As shown in Fig. 1A, the level was much lower in the HT29 and HCT116 cells when compared with that in the SW480, SW620, SW1116 and DLD1 cells. Therefore, HT29 and HCT116 cells were applied in our further experiments.

Next, we incubated HT29 and HCT116 cells with DFX at doses of $0,50,100$ and $200 \mu \mathrm{M}$, respectively, for $48 \mathrm{~h}$, to determine the maximum efficacy at inducing EMT in both cell lines. Treatment with DFX resulted in marked HIF-1 $\alpha$ expression in a dose-dependent manner at the range of 0 to $100 \mu \mathrm{M}$ as shown in Fig. 1B. No significant difference between cells was noted at 100 and $200 \mu \mathrm{M}$. Thus, the concentration of $100 \mu \mathrm{M}$ was used in the following experiments.

DFX treatment enhances cell adhesion, migration and invasion abilities. Considering that the adhesion of cancer cells to the extracellular matrix (ECM) is the first step in tumor metastasis $(24,25)$, we first evaluated the effect of DFX on cell adhesion. HT29 and HCT116 cells with or without DFX 

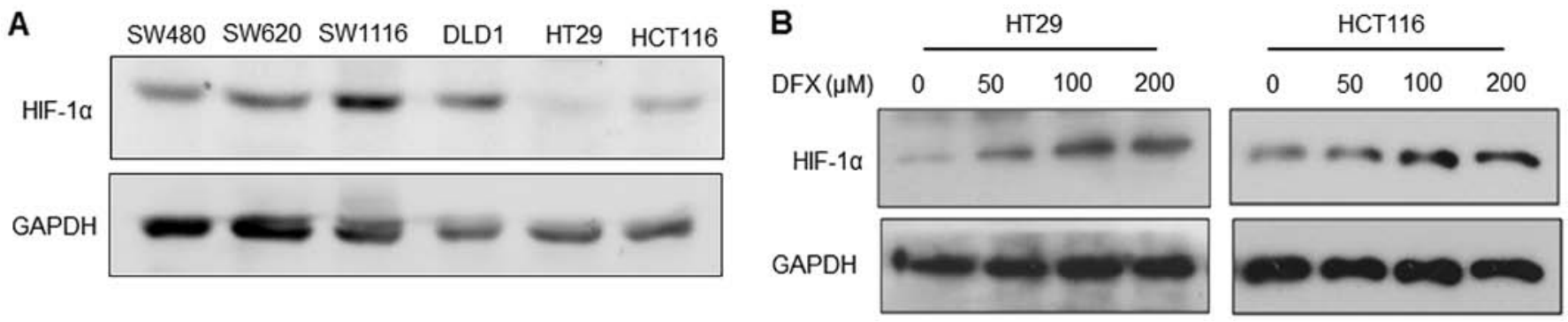

Figure 1. Induction of HIF-1 $\alpha$-expression by DFX in CRC cell lines. (A) Protein expression of HIF-1 $\alpha$ in the indicated 6 cell lines was detected by western blotting. GAPDH was loaded as the internal control. (B) HT29 and HCT116 cells were stimulated with DFX (50, 100 and $200 \mu \mathrm{M})$ or were mock treated. Proteins were loaded $48 \mathrm{~h}$ later and analyzed by western blotting for HIF-1 $\alpha$ expression. GAPDH was used as a loading control. HIF-1 $\alpha$, hypoxia-inducible factor-1 $\alpha$; DFX, deferoxamine; CRC, colorectal cancer; GAPDH, glyceraldehyde-3-phosphate dehydrogenase.
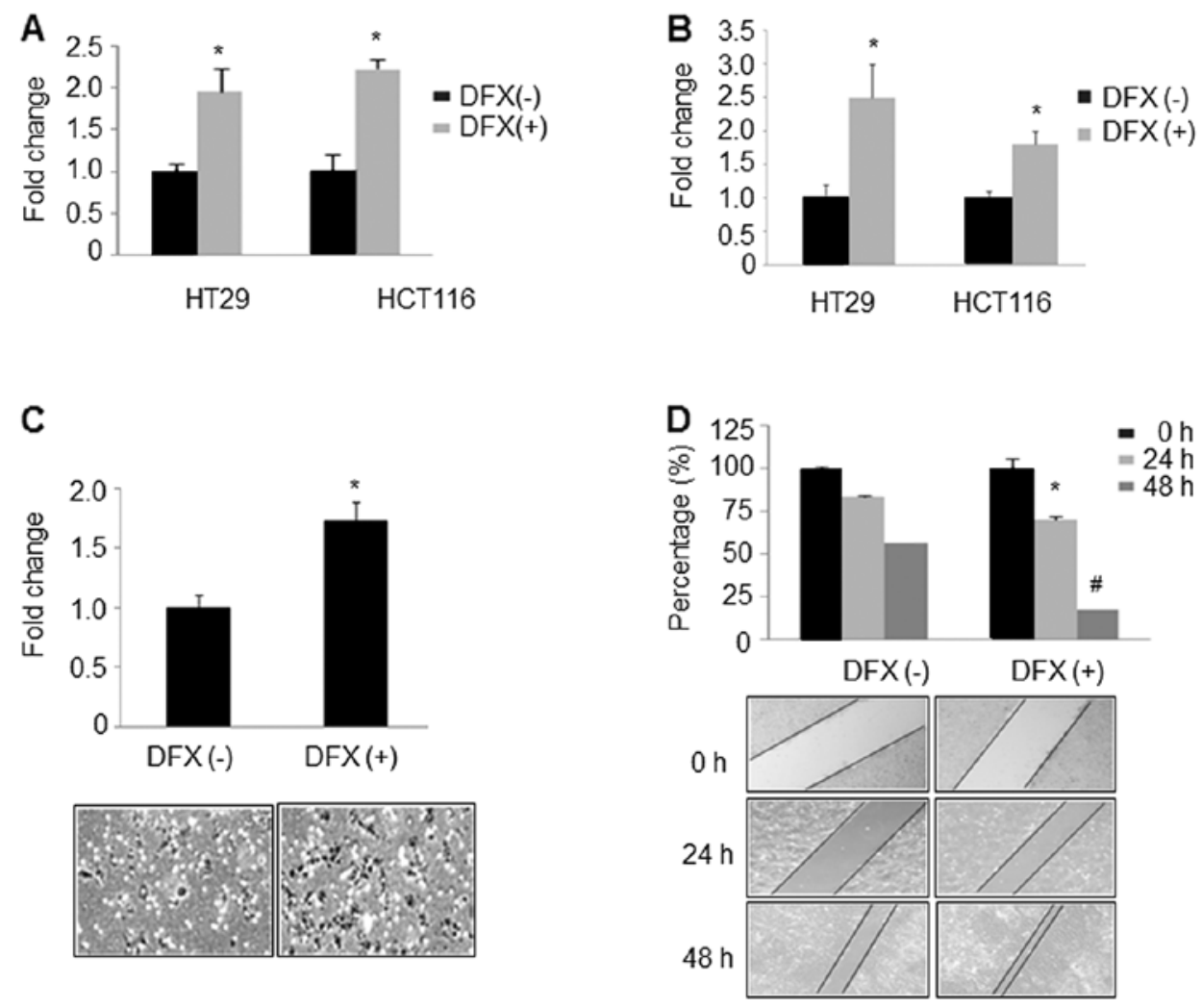

Figure 2. Functional assays of DFX in HT29 and HCT116 cells. (A) Cells were exposed to DFX (100 $\mu \mathrm{M}$ ) or were mock treated for $48 \mathrm{~h}$. Single-cell suspensions were respectively seeded into fibronectin-coated 96 -well plates $\left(5 \times 10^{4} /\right.$ well) for $2 \mathrm{~h}$ followed by quantitative assessment of cell viability by MTT assay. ${ }^{*} \mathrm{P}<0.05$ between comparison groups. (B and C) Migration and invasion assays were performed using Transwell inserts without or with Matrigel. The same number of cells with or without DFX treatment was seeded into the upper compartments of Transwell inserts with FBS-free medium, and the lower compartments were filled with 10\% FBS medium. Quantitative analysis was carried out to determine the relative numbers of cells that crossed the pore filter of the inserts to the bottom medium. (D) Cells treated with DFX were wounded followed by further culturing for another $48 \mathrm{~h}$, then the wound areas were observed at the indicated time points. The relative wound area was calculated (wound area at 24 or $48 \mathrm{~h} /$ wound area of $0 \mathrm{~h}$ ), which was then normalized to that of the DFX-free cells. Data shown are representative of 3 independent experiments; means $\pm \mathrm{SD}$. ${ }^{*} \mathrm{P}<0.05$ comparing with $24 \mathrm{~h}$ and ${ }^{~} \mathrm{P}<0.05$ comparing with $48 \mathrm{~h}$ without $\mathrm{DFX}$ treatment. DFX, deferoxamine; FBS, fetal bovine serum.

treatment were incubated with one major ECM component in fibronectin-coated 96-well plates for $2 \mathrm{~h}$. We found that DFX treatment increased cancer cell adhesion to fibronectin by 1.96- and 2.23-fold in HT29 and HCT116 cells, respectively, when compared to the cells without DFX treatment (Fig. 2A). Next, we detected the effect of DFX on cell migration and invasion. As shown in Fig. 2B and C, DFX significantly increased migration and invasion of HT29 $(2.5 \pm 0.5$ vs. $1 \pm 0.2 ; \mathrm{P}<0.05)$ and HCT116 cells $(1.8 \pm 0.2$ vs. $1 \pm 0.1, \mathrm{P}<0.05)$, when compared with the untreated control cells. Similarly, the wound healing assay also indicated that DFX significantly induced cell migration at 24 and $48 \mathrm{~h}$, respectively (Fig. 2D, $\mathrm{P}<0.05$ ). Collectively, these observations indicate that DFX induced cell adhesion, migration and invasion abilities in HT29 and HCT116 CRC cells.

DFX treatment results in altered cell morphology and cell growth. Morphological change plays an important role in many cellular processes such as migration, differentiation and apoptosis $(22,23,26)$. We then investigated whether the 
A
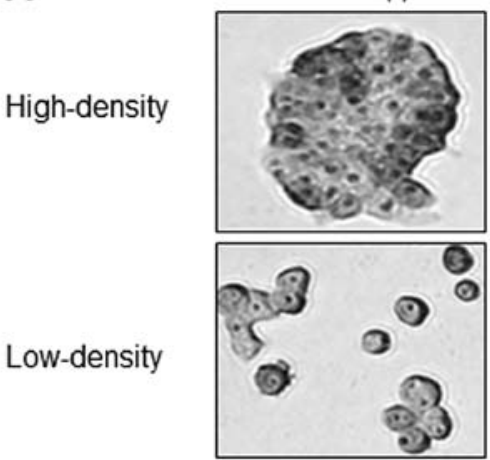

B

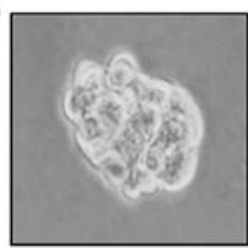

$\operatorname{DFX}(-)$

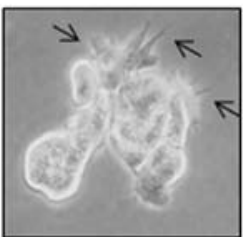

$\operatorname{DFX}(+)$
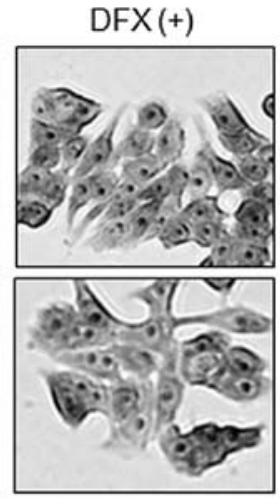

$200 x$

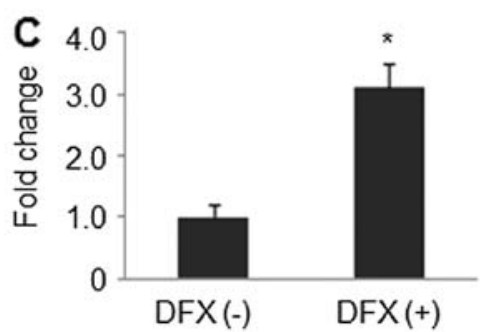

Figure 3. Effect of DFX on cell morphology and cell growth. (A) Representative examples of light microscopic images of H\&E-stained HT29 cells without or with DFX treatment in high- and low-density fields, respectively. Original magnification, $\mathrm{x} 200$. Colony formation assay was performed by observing (B) sphere morphology and (C) calculating the number of spheres. Arrows indicate finger-like protrusions in $\mathrm{B}$. ( $\mathrm{P}<0.05)$. The samples were measured in triplicates, and the experiment was independently performed 3 times. DFX, deferoxamine; H\&E, hematoxylin and eosin.

A

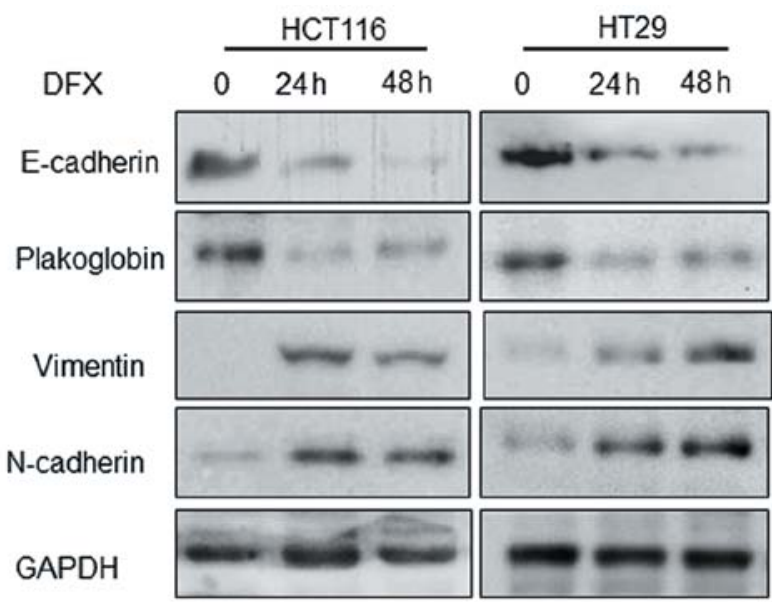

B

E-cadherin

Vimentin

Merge
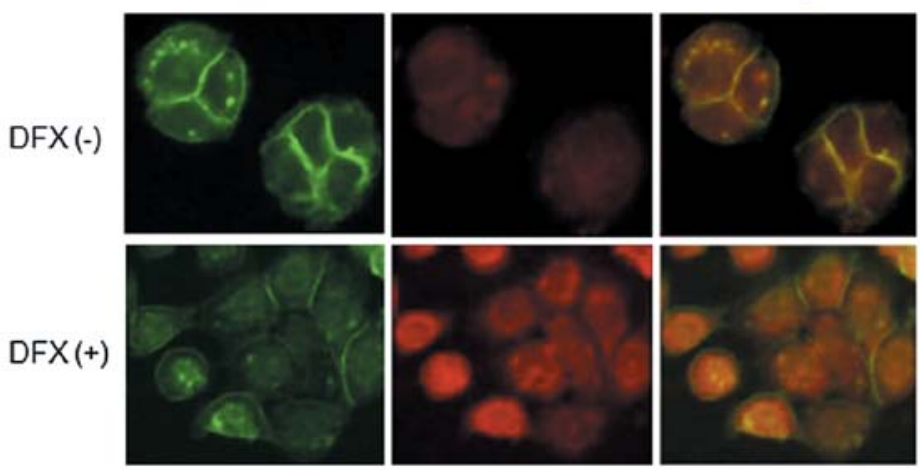

Nucleus

$\operatorname{DFX}(+)$

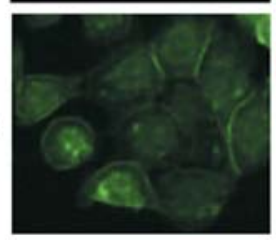

Figure 4. DFX alters the expression and localization of EMT markers. (A) HT29 and HCT116 cells were exposed to DFX for 24 or 48 h, respectively. E-cadherin, plakoglobin, vimentin and N-cadherin expression was detected by western blot analysis with GAPDH used as the internal control. (B) Membraneassociated E-cadherin and vimentin were assessed with the corresponding primary antibodies, and Texas Red- or FITC-labeled secondary antibodies. Cell nuclei are shown by Hoechst 33258 staining. Representative images of immunofluorescence staining are shown at an original magnification of x200. DFX, deferoxamine; EMT, epithelial-mesenchymal transition; GAPDH, glyceraldehyde-3-phosphate dehydrogenase. 
increased motility and invasive ability of CRC cells was coupled with any morphological change. H\&E staining results revealed that treatment with DFX resulted in dramatic morphological alterations in HT29 cells at both high-density and low-density as shown in Fig. 3A. In fact, cells treated with DFX displayed flattened, spread morphology. Cells became more isolated and looked like spindle-shaped and fibroblast-like cells. In contrast the untreated HT29 cells were much more round and had very tight connections. Similar results were found in soft agar assay experiments. As shown in Fig. 3B, cell-cell contacts through the 'pseudopod' were present in DFX-treated cells. The edges of these cells commonly showed several finger-like protrusions which may aid in cell motility. In contrast, cells without DFX treatment had a clear boundary and resembled scattered spheres. At the same time, DFX significantly increased anchorage-independent cell growth by 3.1-fold (Fig. 3C).

Effect of DFX on EMT markers. In addition to alterations in the functions (adhesion, migration and invasion) and cell morphology, the altered expression of EMT markers is another typical feature of EMT and cancer metastasis. We evaluated the effect of DFX on EMT epithelial markers (E-cadherin and plakoglobin) and mesenchymal markers (vimentin and $\mathrm{N}$-cadherin). Western blot analysis indicated that E-cadherin and plakoglobin expression was significantly decreased, while vimentin and $\mathrm{N}$-cadherin expression was increased after DFX treatment for 24 or 48 h (Fig. 4A). As a critical adhesion molecular, E-cadherin is reported as the most important epithelial marker of EMT. We then observed the expression and localization of E-cadherin and vimentin in cells with or without DFX treatment by immunofluorescence staining. As shown in Fig. 4B, the expression of E-cadherin in the cell membrane was markedly decreased in the DFX-treated HT29 cells, which indicated lower cell adhesion ability. At the same time, vimentin expression was upregulated. Furthermore, DFX-treated cells were able to spread much wider on coverslips which was consistent with the H\&E staining results (Fig. 3A). These findings suggest that DFX treatment promotes EMT progression in CRC.

\section{Discussion}

Accumulating evidence indicates that hypoxia or overexpression of HIF-1 $\alpha$ are linked to the genesis, progression and metastasis of human cancers, suggesting HIF-1 $\alpha$ as an emerging biomarker for cancer prognosis and as a promising therapeutic target (27-29). Using a DFX-induced hypoxia model, we revealed that DFX promoted HIF-1 $\alpha$ expression and enhanced cell adhesion, migration and invasion abilities in CRC cells, through modulating cell morphology and expression of EMT markers.

Hypoxia is a major pathophysiological condition for the induction of angiogenesis, which is a common and crucial aspect of cell growth in solid tumors (10-12,30). DFX-induced hypoxia is a complicated event, which may affect a variety of cellular pathways and gene expression. Particularly, DFX is capable of inducing the activation of HIF-1 $\alpha$ and NF- $\mathrm{BB}$ (31). HIF- $1 \alpha$ represents the most important transcription factor regulating gene expression under hypoxic conditions. It acts by binding to hypoxia-responsive elements (HREs) in promoters, thereby regulating gene expression and playing a central role in cancer tumors. Yang et al (20) reported that HIF-1 $\alpha$ promotes tumor progression, EMT and metastasis by direct regulation of Twist, a key transcriptional regulator of EMT. In the present study, we found that DFX increased the HIF-1 $\alpha$ protein level in a dose-dependent manner, which may be one of the mechanisms of DFX-induced cell migration and invasion.

As an important adhesion molecule, membrane E-cadherin is an important hallmark of EMT $(15,19,32)$. We demonstrated that incubation of HT29 cells with DFX led to the loss of E-cadherin in the cell membrane. Furthermore, the expression of EMT mesenchymal marker, vimentin, was increased following DFX treatment, which are both consistent with the EMT event.

In conclusion, the present study demonstrated that DFX increased HIF-1 $\alpha$ protein, decreased membrane-associated E-cadherin expression and increased vimentin expression. Consequently, less E-cadherin combines with other adhesion molecules on the membrane to decrease cell-cell contact and increase migration and invasion. At the same time, due to the transition from an epithelial-like to a mesenchymal-like cell morphology, cell motility was facilitated. These findings explain, in part, the marked role of DFX in colorectal cancer metastasis.

\section{Acknowledgements}

The present study was supported by the National Natural Science Foundation of China (nos. 81172057 and 81272761), the 'President Foundation of Nanfang Hospital, Southern Medical University' (2012B009), and a 'high-level topic matching funds' of Nanfang Hospital (G201227).

\section{References}

1. Kalinowski DS and Richardson DR: The evolution of iron chelators for the treatment of iron overload disease and cancer. Pharmacol Rev 57: 547-583, 2005.

2. Mantovani A, Evans R and Alexander P: Non-specific cytotoxicity of spleen cells in mice bearing transplanted chemically induced fibrosarcomas. Br J Cancer 36: 35-40, 1977.

3. Boult J, Roberts K, Brookes MJ, et al: Overexpression of cellular iron import proteins is associated with malignant progression of esophageal adenocarcinoma. Clin Cancer Res 14: 379-387, 2008.

4. Miller LD, Coffman LG, Chou JW, et al: An iron regulatory gene signature predicts outcome in breast cancer. Cancer Res 71: 6728-6737, 2011.

5. Follézou JY and Bizon M: Cancer chemotherapy induces a transient increase of serum-iron level. Neoplasma 33: 225-231, 1986.

6. Yu Y, Gutierrez E, Kovacevic Z, et al: Iron chelators for the treatment of cancer. Curr Med Chem 19: 2689-2702, 2012.

7. Recalcati S, Locati M, Gammella E, Invernizzi P and Cairo G: Iron levels in polarized macrophages: regulation of immunity and autoimmunity. Autoimmun Rev 11: 883-889, 2012.

8. Kaczmarek M, Cachau RE, Topol IA, Kasprzak KS, Ghio A and Salnikow K: Metal ions stimulated iron oxidation in hydroxylases facilitates stabilization of HIF-1 $\alpha$ protein. Toxicol Sci 107: 394-403, 2009.

9. Martínez-Romero R, Martínez-Lara E, Aguilar-Quesada R, Peralta A, Oliver FJ and Siles E: PARP-1 modulates deferoxamine-induced HIF-1 $\alpha$ accumulation through the regulation of nitric oxide and oxidative stress. J Cell Biochem 104: 2248-2260, 2008.

10. Yang Y, Sun M, Wang L and Jiao B: HIFs, angiogenesis, and cancer. J Cell Biochem 114: 967-974, 2013. 
11. Simiantonaki N, Taxeidis M, Jayasinghe C, Kurzik-Dumke U and Kirkpatrick C: Hypoxia-inducible factor 1 alpha expression increases during colorectal carcinogenesis and tumor progression. BMC Cancer 8: 320, 2008.

12. Semenza GL: Hypoxia, clonal selection, and the role of HIF-1 in tumor progression. Crit Rev Biochem Mol Biol 35: 71-103, 2000.

13. Vincent-Salomon A and Thiery JP: Host microenvironment in breast cancer development: epithelial-mesenchymal transition in breast cancer development. Breast Cancer Res 5: 101-106, 2003.

14. Zhang W, Jiang B, Guo Z, et al: Four-and-a-half LIM protein 2 promotes invasive potential and epithelial-mesenchymal transition in colon cancer. Carcinogenesis 31: 1220-1229, 2010.

15. Zhang W, Wang J, Zou B, et al: Four and a half LIM protein 2 (FHL2) negatively regulates the transcription of E-cadherin through interaction with Snail1. Eur J Cancer 47: 121-130, 2011.

16. Kahlert UD, Nikkhah G and Maciaczyk J: Epithelial-tomesenchymal(-like) transition as a relevant molecular event in malignant gliomas. Cancer Lett 331: 131-138, 2013.

17. Roy N, Bommi PV, Bhat UG, et al: DDB2 suppresses epithelialto-mesenchymal transition in colon cancer. Cancer Res 73 : 3771-3782, 2013

18. Higgins DF, Kimura K, Bernhardt WM, et al: Hypoxia promotes fibrogenesis in vivo via HIF-1 stimulation of epithelial-to-mesenchymal transition. J Clin Invest 117: 3810-3820, 2007.

19. Jiang J, Tang YL and Liang XH: EMT: a new vision of hypoxia promoting cancer progression. Cancer Biol Ther 11: 714-723, 2011.

20. Yang $\mathrm{MH}, \mathrm{Wu} \mathrm{MZ}$, Chiou $\mathrm{SH}$, et al: Direct regulation of TWIST by HIF-1 $\alpha$ promotes metastasis. Nat Cell Biol 10: 295-305, 2008

21. Zhang $\mathrm{Q}$, Bai $\mathrm{X}$, Chen $\mathrm{W}$, et al: Wnt/ $\beta$-catenin signaling enhances hypoxia-induced epithelial-mesenchymal transition in hepatocellular carcinoma via crosstalk with hif-1 $\alpha$ signaling. Carcinogenesis 34: 962-973, 2013.
22. Wang J, Yang Y, Xia HH, et al: Suppression of FHL2 expression induces cell differentiation and inhibits gastric and colon carcinogenesis. Gastroenterology 132: 1066-1076, 2007.

23. Hollestelle A, Peeters JK, Smid M, et al: Loss of E-cadherin is not a necessity for epithelial to mesenchymal transition in human breast cancer. Breast Cancer Res Treat 138: 47-57, 2013.

24. Lester BR and McCarthy JB: Tumor cell adhesion to the extracellular matrix and signal transduction mechanisms implicated in tumor cell motility, invasion and metastasis. Cancer Metastasis Rev 11: 31-44, 1992.

25. Bartolazzi A, Cerboni C, Nicotra MR, Mottolese M, Bigotti A and Natali PG: Transformation and tumor progression are frequently associated with expression of the alpha 3/beta 1 heterodimer in solid tumors. Int J Cancer 58: 488-491, 1994.

26. Wei L, Yang Y, Zhang X and Yu Q: Altered regulation of Src upon cell detachment protects human lung adenocarcinoma cells from anoikis. Oncogene 23: 9052-9061, 2004.

27. Zheng Y, Ni Y, Huang X, Wang Z and Han W: Overexpression of HIF-1 $\alpha$ indicates a poor prognosis in tongue carcinoma and may be associated with tumour metastasis. Oncol Lett 5: 1285-1289, 2013.

28. Cheli Y, Giuliano S, Fenouille N, et al: Hypoxia and MITF control metastatic behaviour in mouse and human melanoma cells. Oncogene 31: 2461-2470, 2012.

29. Martinez-Outschoorn UE, Trimmer C, Lin Z, et al: Autophagy in cancer associated fibroblasts promotes tumor cell survival: role of hypoxia, HIF1 induction and NFKB activation in the tumor stromal microenvironment. Cell Cycle 9: 3515-3533, 2010.

30. Tanaka $T$ and Nangaku M: Angiogenesis and hypoxia in the kidney. Nat Rev Nephrol 9: 211-222, 2013.

31. Haddad JJ, Olver RE and Land SC: Antioxidant/pro-oxidant equilibrium regulates HIF- $1 \alpha$ and $N F-\kappa B$ redox sensitivity. Evidence for inhibition by glutathione oxidation in alveolar epithelial cells. J Biol Chem 275: 21130-21139, 2000.

32. Schmalhofer O, Brabletz $S$ and Brabletz T: E-cadherin, $\beta$-catenin, and ZEB1 in malignant progression of cancer. Cancer Metastasis Rev 28: 151-166, 2009. 